\title{
A 3D GBSM for High-Speed Train Communication Systems Under Deep Cutting Scenarios
}

\author{
Liu Feng*, Pingzhi Fan*, Chengxiang Wang ${ }^{\dagger}$, Ammar Ghazal $^{\dagger}$ \\ * Institute of Mobile Communications, Southwest Jiaotong University, Chengdu, China \\ $\dagger$ Institute of Sensors, Signal and System, School of Engineering and Physical Sciences, \\ Heriot-Watt University, Edinburgh EH14 4AS, UK \\ Email: fengliuswjtu@163.com, p.fan@ieee.org, \{cheng-xiang.wang, ag289\}@hw.ac.uk
}

\begin{abstract}
This paper proposes a novel three-dimensional (3D) cylinder geometry-based stochastic model (GBSM) for nonisotropic multiple-input multiple-output (MIMO) Rice fading channels in high-speed train (HST) wireless communications under deep cutting scenarios. Using a validated approximation, the closed-form expression of the space-time correlation function (ST CF) of the proposed GBSM is obtained. Different from twodimensional (2D) channel models, in the 3D GBSM the elevation angles and the height of the base station (BS) antenna relative to the mobile station (MS) one are introduced.The numerical results show the rationality of the approximation and how the arrangements of antennas affect the ST CF.

Index Terms-Three dimensional cylinder model, cutting scenario, channel modeling, high speed railway, multiple-input multiple-output
\end{abstract}

\section{INTRODUCTION}

Channel modeling for HST wireless communication systems has attracted great interests recently, due to the rapid development of HSTs. HSTs can operate across many scenarios during its travel, such as viaduct, cutting (or U-shape groove), tunnel, etc. Cutting, as one of the most common scenarios of HST communications, is used for ensuring the train's high speed travel and the smoothness of rails. It is usually built on uneven round or "cut" through hills. The depths and slopes of the walls on both sides of the rail are almost the same [1] Cutting can be further divided into two kinds: deep cutting and low cutting, depending on whether the upper eave of the cutting is higher than the antennas of the train or not. In this paper, we focus on the deep cutting scenario, as the deep cutting results in lots of reflection and scattering from the walls, therefore the propagation characteristics are very different from other HST scenarios. Fig. 1 shows a detailed structure of a deep cutting scenario.

Although some measurement campaigns [1]-[4] have been conducted for cutting scenarios, little work on channel modeling of cutting has been undertaken. The existing well-known models such as WINNER II [5] and LTE-R [6], do not cover cutting scenarios. In [7], a one-ring model was proposed for HST channels in cutting scenarios. The scatterers were assumed to be distributed on a ring around the MS. In [8], [9], ellipse models were introduced, where time-varying model parameters and non-stationarity characters were considered. However, all the aforementioned models for HST channels

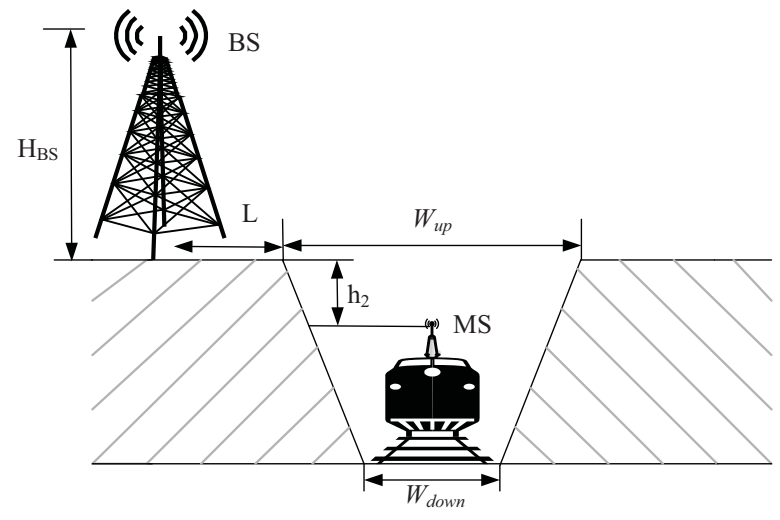

Fig. 1. The geometry of a deep cutting HSR scenario

assumed that the waves are traveling from BS to MS in the horizontal plane only. This assumption is valid for rural or viaduct but it is impractical for the deep cutting scenario where the trains' antenna arrays are located close to and lower than the walls of the cutting. Scattered waves may propagate by diffraction from both side walls, up or down to the antennas of the train and thus not travel in the horizontal plane only. Consequently, 3D channel models are needed.

A channel model for cutting based on 3D ray-tracing was proposed in [10]. The scatterers were modeled by rectangular boxes, where the dimensions of the boxes were generated statistically. As the channel parameters are described in a completely deterministic way, the model is accurate but very complicated. To avoid such a high complexity without a significant accuracy loss, a semi-deterministic model for cutting was proposed in [11], where only large-scale fading was considered and small-scale fading was neglected. In [12], a 3D one-sphere model was introduced to describe the scatterers in urban and suburb scenarios of HST systems. The model of cutting in the paper was also generated in a deterministic way. To the best of the authors' knowledge, there is no regular-shaped GBSM (RS-GBSM) for HST cutting scenarios yet. RS-GBSM has widely been used for channel modeling due to the convenience for theoretical analysis of channel statistics [13]. In this paper, based on the characteristics of deep cutting, a 3D RS-GBSM is proposed. 
Vertical cylinder [14]-[16] is chosen to model the scattering surfaces and diffracting edges of the deep cutting. Though the steep walls are not straight vertical surfaces, walls are usually covered by grass and reinforced concrete, where diffuse reflectance occurs. Compared with the distance between BS and MS, the length of the slope is very small. Both sides of walls have the same depths and slopes, making a "canyon" form. Cylinder has been proved efficiently to model a "canyon" [15].

The rest of paper is organized as follows. The details of the proposed 3D model are described in Section II. The ST $\mathrm{CF}$ of the model is derived in Section III. Section IV presents the numerical results. Finally, some concluding remarks are highlighted in Section V.

\section{The Three Dimensional Channel Model}

Considering a downlink MIMO channel, the numbers of BS antennas and MS antennas are denoted by $L_{T}$ and $L_{R}$, respectively. The number of antenna elements are $1 \leq p \leq$ $p^{\prime} \leq L_{T}$ and $1 \leq q \leq q^{\prime} \leq L_{R}$. Here, we take a $2 \times 2$ MIMO channel as an example.

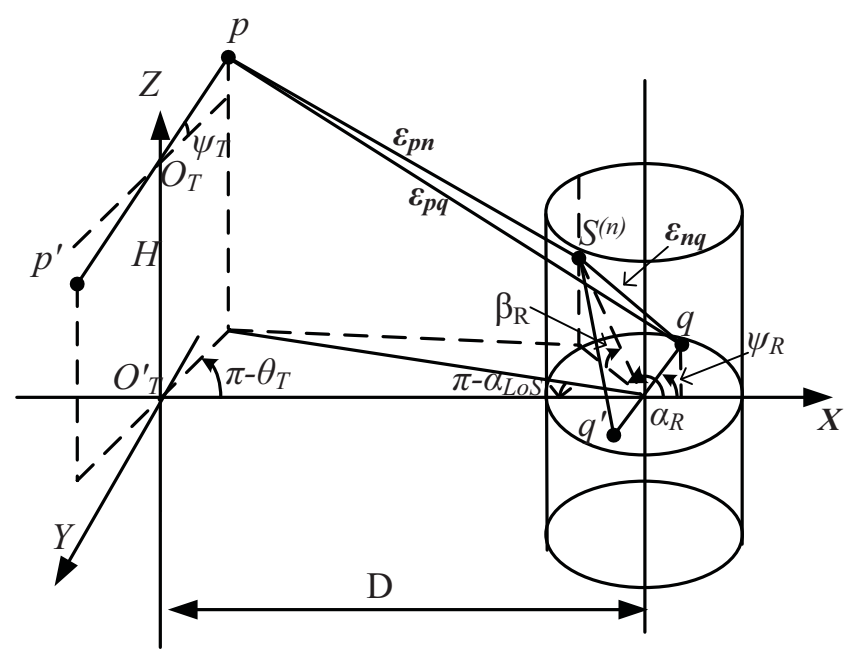

Fig. 2. The 3D cylinder GBSM for a HST communication system under deep cutting scenarios.

Fig. 2 shows the proposed model with the geometry of lineof-sight (LoS) and the single-bounce (SB) components. There are $N$ effective local scatterers lying on the lateral surface of a cylinder with radius $R$ around the MS and the $n$th scatterer is denoted by $S^{(n)}$. BS antennas are free of local scatters since they are high enough. The parameters in Fig. 2 are defined in Table I.

Based on the GBSM described in Fig. 2, the channel impulse response (CIR) $h_{p q}$ of the channel is a superposition of the LoS and non-LoS (NLoS) rays. The CIR can be expressed as

$$
h_{p q}(t)=h_{p q}^{L o S}(t)+h_{p q}^{S B}(t) .
$$

For LoS component,

$h_{p q}^{L o S}(t)=\Omega_{p q} \sqrt{\frac{K}{K+1}} e^{-j \frac{2 \pi}{\lambda} \varepsilon_{p q}} e^{j 2 \pi t f_{\max } \cos \left(\alpha^{L o S}-\gamma_{R}\right)}$.

For NLoS components,

$$
\begin{aligned}
h_{p q}^{S B}(t) & =\Omega_{p q} \sqrt{\frac{1}{(K+1) N}} \\
& \times \lim _{N \rightarrow \infty} \sum_{n=1}^{N} e^{j\left(\psi_{n}-\frac{2 \pi}{\lambda} \varepsilon_{p q, n}\right)} \\
& \times e^{j 2 \pi f_{\max } t \cos \left(\alpha_{R}^{(n)}-\gamma_{R}\right) \cos \left(\beta_{R}^{(n)}\right)}
\end{aligned}
$$

where $\Omega_{p q}$ denotes the power transmitted through the subchannel $p q, K$ is the Rice factor of the sub-channel $p q, \lambda$ is the carrier wavelength and $f_{\max }$ is the maximum Doppler frequency of MS. The phase $\psi_{n}$ is a random variable, uniformly distributed in the interval $[-\pi, \pi)$ and independent from angles of arrival (AoA) [14].

The distances $\varepsilon_{p q}, \varepsilon_{p n}, \varepsilon_{n q}$, which are functions of the random variables $\alpha_{L o S}, \alpha_{R}^{(n)}$, and $\beta_{R}^{(n)}$, can be expressed as

$$
\begin{gathered}
\varepsilon_{p q} \approx \frac{H}{\sin \beta_{B}}-\frac{L_{T}+1-2 p}{2 \cos \beta_{B}} \delta_{T} \cos \theta_{T} \cos \psi_{T} \\
\left.-\frac{L_{R}+1-2 q}{2 \cos \beta_{B}} \delta_{R} \cos \psi_{R} \cos \left(\alpha^{L o S}-\theta_{R}\right)\right) \\
\varepsilon_{p n} \approx \frac{H}{\sin \beta_{B}}-\frac{\left(L_{T}+1-2 p\right) \delta_{T} \cos \psi_{T}}{2 \cos \beta_{B}} \\
\quad \times\left(\cos \theta_{T}+\Delta \sin \theta_{T} \sin \alpha_{R}^{(n)}\right)
\end{gathered}
$$

TABLE I

DEFINITIONS OF THE KEY PARAMETERS USED IN THE CYLINDER GBSM IN FIG. 2.

\begin{tabular}{|c|l|}
\hline$D$ & $\begin{array}{l}\text { The distance between the BS and } \\
\text { the centers of MS cylinders. }\end{array}$ \\
\hline$R$ & The radius of the MS cylinder. \\
\hline$\delta_{T}, \delta_{R}$ & Antenna element spacings of MS and BS \\
\hline$\theta_{T}, \theta_{R}$ & $\begin{array}{l}\text { Orientation of the BS } \\
\text { and MS antenna array. }\end{array}$ \\
\hline$\psi_{T}, \psi_{R}$ & $\begin{array}{l}\text { Elevation of the BS } \\
\text { and MS antenna array }\end{array}$ \\
\hline$\alpha^{L o S}, \alpha_{R}^{(n)}$ & $\begin{array}{l}\text { Azimuth angle of arrival (AAoA) of the LoS and } \\
\text { of the wave scattered from } S^{(n)}\end{array}$ \\
\hline$H$ & $\begin{array}{l}\text { The height of the BS antenna elements relative to } \\
\text { the one of the MS antenna elements }\end{array}$ \\
\hline$\beta_{R}^{(n)}$ & $\begin{array}{l}\text { Elevation angles of arrival (EAoA) } \\
\text { of the waves scattered from } S^{(n)}\end{array}$ \\
\hline$\varepsilon_{p q}$ & $\begin{array}{l}\text { Distances between antenna element p and q } \\
\text { Distance between antenna p and the scatter } S^{(n)} \\
\text { distance between the scatter } S^{(n)} \text { and antenna q }\end{array}$ \\
\hline$\varepsilon_{p n}, \varepsilon_{n q}$ & \\
\hline
\end{tabular}




$$
\begin{aligned}
\varepsilon_{n q} & \approx R-\frac{\left(L_{R}+1-2 q\right) \delta_{R}}{2} \\
& \times\left[\cos \psi_{R} \cos \theta_{R} \cos \alpha_{R}^{(n)} \cos \beta_{R}^{(n)}\right. \\
& +\cos \psi_{R} \sin \theta_{R} \sin \alpha_{R}^{(n)} \cos \beta_{R}^{(n)} \\
& \left.+\sin \psi_{R} \sin \beta_{R}^{(n)}\right]
\end{aligned}
$$

where $\beta_{B}$ is the elevation angle of the BS relative to the $x-y$ plane, $\beta_{B}=\arctan (H / D)$. Note that $\Delta \approx$ $\arcsin (R / D)$. Here, $R / D$ is a narrow angle of spread, when $\max \left\{\delta_{T}, \delta_{R}\right\}<<R<<D$. The approximations in (4)-(6) are due to small angle approximations, i.e., $\sin \alpha \approx$ aand $\cos \alpha \approx$ 1 for small $\alpha$ [14].

\section{Statistical Properties of the Proposed GBSM}

\section{A. Normalized ST CF}

Under the wide-sense stationary uncorrelated scattering (WSSUS) condition, the ST CF between two sub-channels $h_{p q}(t)$ and $h_{p^{\prime} q^{\prime}}(t)$ is defined as [15].

$$
\rho_{p q, p^{\prime} q^{\prime}}(\tau)=\frac{E\left\{h_{p q}(t) h_{p^{\prime} q^{\prime}}^{*}(t+\tau)\right\}}{\sqrt{E\left\{\left|h_{p q}(t)\right|^{2}\right\} E\left\{\left|h_{p^{\prime} q^{\prime}}^{*}(t)\right|^{2}\right\}}}
$$

where $E\{*\}$ is the statistical expectation operator and * denotes complex conjugate operation. Since $h_{p q}^{\operatorname{LoS}}(t)$ and $h_{p q}^{S B}(t)$ are independent zero-mean complex Gaussian random processes [13], (7) can be expressed as a summation of the ST CFs of the LoS component $\rho_{p q, p^{\prime} q^{\prime}}^{L o S}(\tau)$ and SB components $\rho_{p q, p^{\prime} q^{\prime}}^{S B}(\tau)$, i.e.,

$$
\rho_{p q, p^{\prime} q^{\prime}}(\tau)=\rho_{p q, p^{\prime} q^{\prime}}^{\operatorname{LoS}}(\tau)+\rho_{p q, p^{\prime} q^{\prime}}^{S B}(\tau) .
$$

As $\max \left\{\delta_{T}, \delta_{R}\right\} \ll D$, we can obtain that $\alpha^{L o S} \approx \pi$ and (4) can be simplified. Then, $\rho_{p q, p^{\prime} q^{\prime}}^{L o S}$ and $\rho_{p q, p^{\prime} q^{\prime}}^{S B}$ can be expressed as

$$
\begin{gathered}
\rho_{p q, p^{\prime} q^{\prime}}^{L o S}\left(\tau, \delta_{T}, \delta_{R}\right)= \\
\quad e^{\frac{j 2 \pi}{\lambda \cos \beta_{B}}\left(\delta_{T} \cos \psi_{T} \cos \theta_{T}-\delta_{R} \cos \psi_{R} \cos \theta_{R}\right)} \\
\times e^{j 2 \pi \tau f_{\max } \cos \gamma_{R}} \\
\rho_{p q, p^{\prime} q^{\prime}}^{S B}\left(\tau, \delta_{T}, \delta_{R}\right)= \\
\quad \lim _{N \rightarrow \infty} \sum_{n=1}^{N} E\left\{e^{-j 2 \pi \tau f_{\max } \cos \left(\alpha_{R}^{(n)}-\gamma_{R}\right) \cos \left(\beta_{R}^{(n)}\right)}\right. \\
\times e^{\frac{j 2 \pi \delta_{T} \cos \psi_{T}}{\lambda \cos \beta_{B}}\left(\Delta \sin \left(\theta_{T}\right) \sin \alpha_{R}^{(n)}+\cos \theta_{T}\right)} \\
\times e^{\frac{j 2 \pi \delta_{R}}{\lambda} \cos \psi_{R} \cos \beta_{R}^{(n)}\left(\cos \alpha_{R}^{(n)} \cos \theta_{R}+\sin \alpha_{R}^{(n)} \sin \theta_{R}\right)} \\
\left.\times e^{\frac{j 2 \pi \delta_{R}}{\lambda} \sin \psi_{R} \sin \beta_{R}^{(n)}}\right\}
\end{gathered}
$$

where $\beta_{\max }$ is the maximum elevation angle of random scatterers. Assuming that the scattering environment is nonisotropic, the Von Mises probability density function (PDF) and the Parson PDF are used for describing the scatterer's azimuth and elevation angles of arrival $\alpha_{R}$ and $\beta_{R}$, respectively.

The Von Mises PDF [17] is,

$$
f(\theta)=\exp [k \cos (\theta-\mu)] / 2 \pi I_{0}(k)
$$

where $\theta \in[-\pi, \pi), \mu \in[-\pi, \pi)$ is the mean angle at which the scatterers are distributed in the horizontal plane, $I_{0}(*)$ is the zeroth-order modified Bessel function of the first kind, and $k$ is a positive real value that denotes the spread of scatterers around the mean angle $\mu$. As $k$ increases, the scatterers become more non-isotropic.

The Parson PDF is defined as [18]

$$
y=\left\{\begin{array}{l}
\frac{\pi}{4\left|\varphi_{\max }\right|} \cos \left(\frac{\pi}{2} \frac{\varphi}{\varphi_{\max }}\right), \quad|\varphi|<\left|\varphi_{\max }\right|<\frac{\pi}{2} \\
0, \quad \text { otherwise }
\end{array}\right.
$$

where $\varphi_{\max }$ in the range of $\left[0,20^{\circ}\right]$, is the maximum elevation angle. Such a maximum elevation angle is typical for the "street-canyon" type of propagation [15]. In this paper, the deep cutting is a typical "street-canyon" for the HST, so the Parson PDF is adopted. According to the equality $\int_{-\pi}^{\pi} e^{a \sin (c)+b \cos (c)} d c=2 \pi I_{0}\left(\sqrt{a^{2}+b^{2}}\right)$ [19]. The normalized ST CF of the SB components can then be simplified as

$$
\begin{aligned}
& \rho_{p q, p^{\prime} q^{\prime}}^{S B}\left(\tau, \delta_{T}, \delta_{R}\right)=\int_{-\beta_{\max }}^{\beta_{\max }} \frac{\pi}{4 \beta_{\max }} \frac{I_{0}\left(\sqrt{\left(a_{1}\right)^{2}+\left(b_{1}\right)^{2}}\right.}{I_{0}(k)} \\
& \times \cos \left(\frac{\pi \beta_{R}}{2 \beta_{\max }}\right) e^{j \frac{2 \pi}{\lambda} \delta_{R} \sin \psi_{R} \sin \beta_{R}} \\
& \times e^{j \frac{2 \pi}{\lambda \cos \beta_{B}} \delta_{T} \cos \psi_{T} \cos \theta_{T}} d \beta_{R}
\end{aligned}
$$

with

$$
\begin{aligned}
a_{1}= & -j 2 \pi \tau f_{\max } \sin \gamma_{R} \cos \beta_{R} \\
& +j \frac{2 \pi}{\lambda \cos \beta_{B}} \delta_{T} \cos \psi_{T} \Delta \sin \theta_{T} \\
& +j \frac{2 \pi}{\lambda} \delta_{R} \cos \psi_{R} \sin \theta_{R} \cos \beta_{R}+k \sin \mu \\
b_{1}= & -j 2 \pi \tau f_{\max } \cos \gamma_{R} \cos \beta_{R} \\
& +j \frac{2 \pi}{\lambda} \delta_{R} \cos \psi_{R} \cos \theta_{R} \cos \beta_{R}+k \cos \mu .
\end{aligned}
$$

In (13), the ST CF of SB components has to be evaluated numerically as there is no closed-form solution of the integrals. In deep cutting scenarios, the maximum of $\beta_{R}$ is small enough to use the small angle approximations $\cos \beta_{R} \approx 1$ and $\sin \beta_{R} \approx \beta_{R}$. Consequently, the ST CF in (13) can be approximated as

$$
\begin{aligned}
\rho_{p q, p^{\prime} q^{\prime}}^{S B}\left(\tau, \delta_{T}, \delta_{R}\right) & \approx \frac{I_{0}\left(\sqrt{\left(a_{11}\right)^{2}+\left(b_{11}\right)^{2}}\right.}{I_{0}(k)} \int_{-\beta_{\max }}^{\beta_{\max }} \frac{\pi}{4 \beta_{\max }} \\
& \times \cos \left(\frac{\pi \beta_{R}}{2 \beta_{\max }}\right) e^{j \frac{2 \pi}{\lambda} \delta_{R} \beta_{R} \sin \psi_{R}} \\
& \times e^{j \frac{2 \pi}{\lambda \cos \beta_{B}} \delta_{T} \cos \psi_{T} \cos \theta_{T}} d \beta_{R}
\end{aligned}
$$


with

$$
\begin{aligned}
a_{11}= & -j 2 \pi \tau f_{\max } \sin \gamma_{R} \\
& +j \frac{2 \pi}{\lambda \cos \beta_{B}} \delta_{T} \cos \psi_{T} \Delta \sin \theta_{T} \\
& +j \frac{2 \pi}{\lambda} \delta_{R} \cos \psi_{R} \sin \theta_{R}+k \sin \mu \\
b_{11}= & -j 2 \pi \tau f_{\max } \cos \gamma_{R} \\
& +j \frac{2 \pi}{\lambda} \delta_{R} \cos \psi_{R} \cos \theta_{R}+k \cos \mu .
\end{aligned}
$$

Finally, solving the integrals in (16), we can get an approximated closed-form expression of ST CF as

$$
\begin{aligned}
\rho_{p q, p^{\prime} q^{\prime}}^{S B}\left(\tau, \delta_{T}, \delta_{R}\right) & \approx \frac{I_{0}\left(\sqrt{\left(a_{11}\right)^{2}+\left(b_{11}\right)^{2}}\right)}{I_{0}(k)} \\
& \times \frac{\cos \left(\frac{2 \pi}{\lambda} \beta_{\max } \delta_{R} \sin \psi_{R}\right)}{1-\left(\frac{4 \delta_{R} \beta_{\max } \sin \psi_{R}}{\lambda}\right)^{2}} \\
& \times e^{j 2 \pi \delta_{T} \cos \theta_{T} \cos \psi_{T}} .
\end{aligned}
$$

\section{B. Spatial cross correlation function (CCF)}

We can get the spatial CCF from ST CF by imposing $\tau=0$ in (9) and (13), i.e.,

$$
\begin{aligned}
R_{p q, p^{\prime} q^{\prime}}^{\operatorname{LoS}} & \left(\delta_{T}, \delta_{R}\right)= \\
& e^{\frac{j 2 \pi}{\lambda \cos \beta_{B}}\left(\delta_{T} \cos \psi_{T} \cos \theta_{T}-\delta_{R} \cos \psi_{R} \cos \theta_{R}\right)}
\end{aligned}
$$

$$
\begin{aligned}
& R_{p q, p^{\prime} q^{\prime}}^{S B}\left(\delta_{T}, \delta_{R}\right)=\int_{-\beta_{\max }}^{\beta_{\max }} \frac{\pi}{4 \beta_{\max }} \frac{I_{0}\left(\sqrt{\left(a_{c 1}\right)^{2}+\left(b_{c 1}\right)^{2}}\right.}{I_{0}(k)} \\
& \times \cos \left(\frac{\pi \beta_{R}}{2 \beta_{\max }}\right) e^{j \frac{2 \pi}{\lambda} \delta_{R} \sin \psi_{R} \sin \beta_{R}} \\
& \times e^{j \frac{2 \pi}{\lambda \cos \beta_{B}} \delta_{T} \cos \psi_{T} \cos \theta_{T}} d \beta_{R}
\end{aligned}
$$

with

$$
\begin{aligned}
a_{c 1}= & j \frac{2 \pi}{\lambda \cos \beta_{B}} \delta_{T} \cos \psi_{T} \Delta \sin \theta_{T} \\
& +j \frac{2 \pi}{\lambda} \delta_{R} \cos \psi_{R} \sin \theta_{R} \cos \beta_{R}+k \sin \mu \\
b_{c 1} & =j \frac{2 \pi}{\lambda} \delta_{R} \cos \psi_{R} \cos \theta_{R} \cos \beta_{R}+k \cos \mu .
\end{aligned}
$$

\section{NumericAl Results}

In all the results illustrated in this section, the following model parameters are used, which are obtained based on measurements in cutting scenarios [1]. The BS antennas have a height of $28 \mathrm{~m}$. The MS antennas are $3 \mathrm{~m}$ lower than the edge of the walls of cuttings. So, $H=31 \mathrm{~m}, R=30 \mathrm{~m}$, and $\beta_{\max }=\arctan (3 / 15)$. We compare the approximated ST CF in (19) with the ST CF in (13) numerically to illustrate the validity of the approximation. Fig. 3 shows absolute values of the ST CFs in (13) and (19) with $D=500 \mathrm{~m}$. The results show in general very good agreement between the exact values and the approximate ones, except discrepancy at certain values.

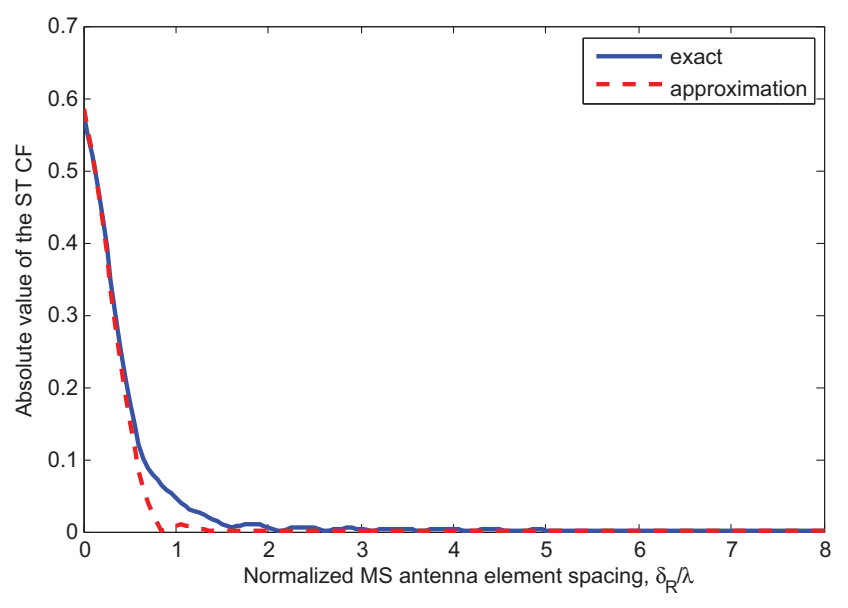

Fig. 3. Absolute values of the ST CF in (13) and (19), assuming the parameters $\psi_{T}=\psi_{R}=\pi / 4, \theta_{T}=\theta_{R}=2 \pi / 3, k=3 \delta_{T}=0.5 \lambda$.

The cylinder model can easily be reduced to a $2 \mathrm{D}$ ring model when $\beta_{R}=0$. Fig. 4 shows the absolute values of the spatial CCFs of the $2 \mathrm{D}$ and $3 \mathrm{D}$ models. It is clear that the spatial CCF of the 3D model is lower than that of the corresponding $2 \mathrm{D}$ model. This is due to the fact that spatial diversity in reality includes both horizontal and vertical parts, while the 2D model ignores the vertical part which leads to an overestimation in the spatial CCF. This agrees with the conclusion reported in [20]

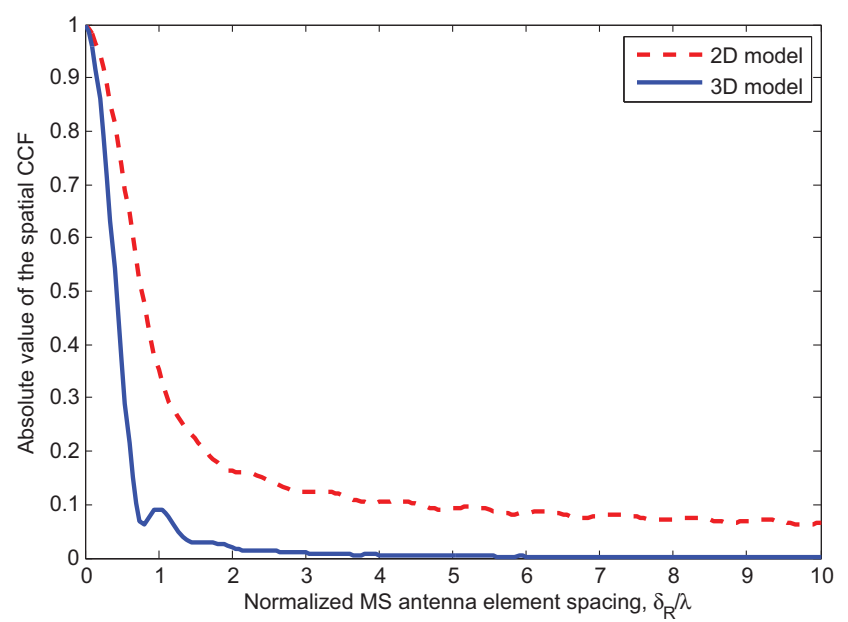

Fig. 4. Absolute values of the CCF, assuming the parameters $\psi_{T}=\psi_{R}=$ $2 \pi / 3, \theta_{T}=\theta_{R}=2 \pi / 3, k=3 \delta_{T}=0.5 \lambda$.

Compared with the 2D model, in the 3D model the elevation angles of BS and MS antenna arrays $\psi_{T}$ and $\psi_{R}$ are introduced. Fig. 5 shows how the ST CF is affected by $\psi_{T}$ and $\psi_{R}$. To elaborate the effects of the elevation angles of both BS and MS antenna arrays, the 3D plot is provided. It is shown that the absolute values of the resulting ST CF decrease rapidly as the elevation angle of MS antenna arrays $\psi_{R}$ increases when $\psi_{R}>0$. The absolute values of the ST CF increase 
as the elevation angle of MS antenna arrays $\psi_{R}$ increases when $\psi_{R}<0$. The absolute values of the ST CF change slowly as the elevation angle of BS antenna arrays changes. Furthermore, we note that the ST CF has a minimal value. We can calculate the minimal value and the corresponding coordinates numerically.

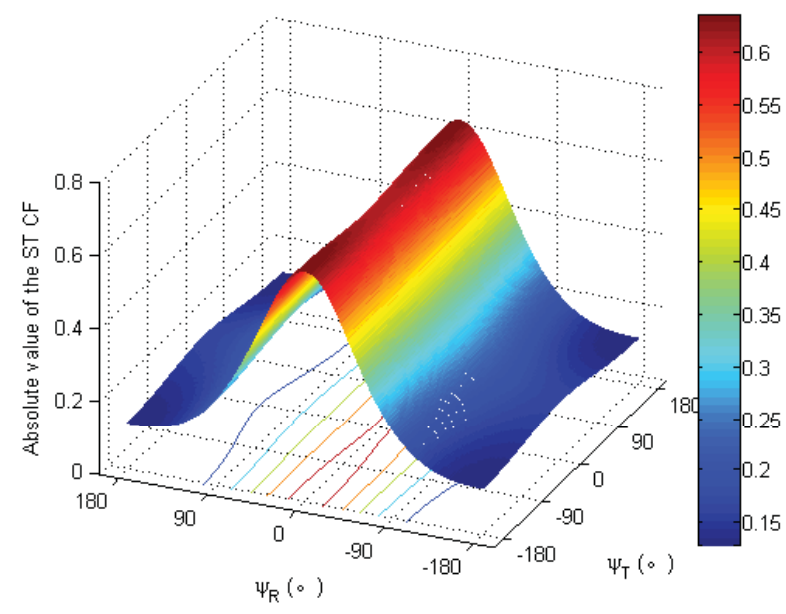

Fig. 5. Absolute values of the ST CF of the elevation of BS and MS antenna arrays, assuming the parameters $\delta_{T}=\delta_{R}=0.5 \lambda, \theta_{T}=\theta_{R}=2 \pi / 3$, and $k=3$.

\section{COnClusions}

A novel 3D RS-GBSM for HST communication systems under deep cutting scenarios has been proposed. Based on the proposed MIMO channel model, the closed-form expression of the ST CF between two arbitrary sub-channels has been derived and analyzed. It has been shown that the $3 \mathrm{D}$ channel model results in lower $\mathrm{CCF}$ values than the corresponding $2 \mathrm{D}$ channel model. Low correlation can be obtained when the BS and MS antennas are carefully designed.

\section{ACKNOWLEDGMENT}

The authors would like to thank the National Basic Research Program of China (973 Program, No. 2012CB316100), the National Science Foundation of China (NSFC, No. 61032002), the 111 Project (No. 111-2-14), the Fundamental Research Funds for the Central Universities (No. SWJTU12ZT02/2682014ZT11, the 863 Project in 5G (No. 2014AA01A707), EU FP7 QUICK project (No. PIRSES-GA2013-612652), and EU H2020 ITN 5G Wireless project (No. 641985)). The authors would also like to acknowledge the support of Ammar Ghazal, Yu Fu, and Cheng-Xiang Wang from Heriot-Watt University, Edinburgh, UK, for sharing the MATLAB implementation of the high-speed train channel simulator.

\section{REFERENCES}

[1] R. He, Z. Zhong, B. Ai, and J. Ding, "Propagation measurements and analysis for high-speed railway cutting scenario," in Electron. Lett., vol. 47 , no. 21 , pp. $1167-1168$, Oct. 2011
[2] J. Lu, G. Zhu, and C. Briso-Rodriguez, "Fading characteristics in the railway terrain cuttings," in Proc. IEEE VTC'11-Spring, Budapest, Hungary, May 2011, pp. 1-5.

[3] R. He, Z. Zhong, B. Ai, J. Ding, and Y. Yang, "Propagation measurements and analysis of fading behavior for high speed rail cutting scenarios," in Proc. IEEE GLOBALCOM'12, California, USA, Dec. 2012, pp. 50155020.

[4] L. Tian, J. Zhang, and C. Pan, "Small scale fading characteristics of wideband radio channel in the U-Shape cutting of high-speed railway," in Proc. IEEE VTC'13-Fall, Las Vegas, USA, Sept. 2013, pp. 1-6.

[5] P. Kyosti et al., "WINNER II channel models," IST-WINNER II D1.1.2, Nov. 2007.

[6] G. Senarth et al., "Multi-hop relay system evaluation methodology (Channel model and performance metric)," IEEE 802.16j-06/013r3, Feb. 2007.

[7] $\mathrm{Q}$. Zheng, C. $\mathrm{Xu}$, and $\mathrm{M}$. Wu, "A novel MIMO channel model for high speed railway system," in Proc. IEEE ICCT'12, Chengdu, China, Nov. 2012, pp. 31-35.

[8] A. Ghazal, C.-X. Wang, H. Haas, M. A. Beach, X. Lu, and D. Yuan, "A non-stationary MIMO channel model for high speed train communication systems," in Proc. IEEE VTC'12-Spring, Yokohama, Japan, May. 2012, pp. 1-5.

[9] A. Ghazal, C.-X. Wang, B. Ai, D. Yuan, and H. Haas, "A non-stationary wideband MIMO channel model for high-mobility intelligent transportation systems," IEEE Trans. Intell. Transp. Syst., vol. 16, no. 2, pp. 885897, Apr. 2015.

[10] S. Knörzer, M. A. Baldauf, T. Fugen, and W. Wiesbeck, "Channel analysis for an OFDM-MISO train communications system using different antennas," in Proc. IEEE VTC'07-Fall, Baltimore, USA, Oct. 2007, pp. 809-813.

[11] K. Guan, Z. Zhong, B. Ai, and T. Kurner, "Semi-deterministic path-loss modeling for viaduct and cutting scenarios of high-speed railway," IEEE Antennas Wireless Propag. Lett, vol. 12, pp. 789-792, June 2013.

[12] B. Chen and Z. Zhong, "Geometry-based stochastic modeling for MIMO channel in high-speed mobile scenario," Int. J. Antennas and Propagation, vol. 2012, Article ID 184682, 6 pages, 2012. doi:10.1155/2012/184682.

[13] Y. Yuan, C.-X. Wang, X. Cheng, B. Ai, and D. I. Laurenson, "Novel 3D geometry-based stochastic models for non-isotropic MIMO vehicleto-vehicle channels", IEEE Trans. Wireless Commun., vol. 13, no. 1, pp. 298-309, Jan. 2014.

[14] E. Michailidis and A. Kanatas, "A three dimensional model for land mobile-HAP-MIMO fading channels," in Proc. Int. Workshop SPSC, Rhodes Island, Greece, Oct. 2008, pp. 1-6.

[15] G. Zajic and G. L. Stuber, "Three-dimensional modeling, simulation, and capacity analysis of space-time correlated mobile-to-mobile channels," IEEE Trans. Veh. Technol., vol. 57, no. 4, pp. 2042-2054, Jul. 2008.

[16] A. G. Zajic and G. L. Stuber, "Three-dimensional modeling and simulation of wideband MIMO mobile-to-mobile channels," IEEE Trans. Wireless Commun., vol. 8, no. 3, pp. 1260-1275, Mar. 2009.

[17] G. L. Stuber, Principle of mobile communication, 2nd Edition, Boston, MA:Kluwer, 2001

[18] J. D. Parsons and A. M. D. Turkmani, "Characterization of mobile radio signals: model description," IEE Proc.-I, vol. 138, no. 6, pp. 549-556, Dec. 1991.

[19] I. S. Gradshteyn and I. M. Ryzhik, Table of Integrals, Series and Products, 5th edition, Edited by A. Jeffrey, Academic Press, 1994.

[20] R. Almesaeed, A. S. Ameen, A. Doufexi, N. Dahnoun and A. R. Nix, "A comparison study of 2D and 3D ITU channel model," in Proc. IEEE Wireless Days, Valencia, Spain, Nov. 2013, pp. 1-7. 